\title{
Subject Hierarchy Structure Modeling in Data Warehouse
}

\author{
Yixuan Ma*, Xuedong Gao and Shujuan Gu \\ Dongling School of Economics and Management, University of Science \\ Technology Beijing, China, 100083 \\ mayixuan0302@gmail.com; gaoxuedong@manage.ustb.edu.cn; \\ gushujuan@ustb.edu.cn
}

\begin{abstract}
Data warehouse is subject-oriented organized. However, when data warehousing, the hierarchy structure of subject is currently only decided by decision makers' intuition. Faced with complicated business data mining cases, it is hard to establish hierarchy structure of subject just according to intuition. Thus a method based on ISM is present to make subject level structure establishment more measurable and illustrative. In this article, the "Subject" level structure establishment process is presented firstly. Then the method is put forward. Finally, the rationality and validity of this method are verified by a case on university financial data warehouse's subject level establishment.
\end{abstract}

Keywords data warehouse $\bullet$ subject level structure $\bullet$ ISM

\section{Introduction}

In data warehouse, data need to be organized and stored according to subject, such as customer, supplier, product, and sales. Rather than concentrating on the day-to-day operations and transaction processing of an organization, a data warehouse focuses on the modeling and analysis of subject-oriented data. In this way, data warehouses typically provide a straightforward and concise view about particular subject issues by excluding data that are not useful in the decision support process. However, currently, subjects are determined by decision makers' intuition. When facing a highly complicated subject system, decision makers are hard to offer a clear and organized level structure of subject in data warehouse just only by their experience.

Interpretative Structural Modeling is defined as a process focused on assisting the human being to comprehend better about what he/she believes and to recognize clearly what he/she does not know [1]. It helps to impose order and direction on the complexity of relationships among various elements of complex system [2]. ISM describes the structure of the system, which describes the relationship between each part and them with the external environment. The so-called relationship includes causality, order, contact relations, and subordinate relations, etc. It is briefness for it just despites the relationship, not involving the size of the quantity. It transits from the concept model of system to the intermediary of quantitative analysis. What most amazing is that it can establish the structure model for systems those hard to quantify. Hence, it is widely used in system analysis and system integrated.

In this paper, the subject level partition algorithm based on Interpretative Structural Modeling (ISM) is proposed to transform unclear and poorly articulated mental models of system into visible and well-defined subject level structure. 


\section{Establish Subject Level Structure Based on ISM}

\subsection{Principle of ISM Method}

Interpretative Structural Modeling (ISM) is a kind of method proposed by J. Warfield for analysis of complex social and economic system in 1973 in USA. ISM is a methodology that aids at identifying a structure within a system [3]. It helps to impose order and direction on the complexity of relationships among various elements of a system [4]. For many tangle problems under consideration, an amount of factors might be related to a problem. However, the situation described by the direct and indirect relationships among the factors is far more accurately than the just isolate factor. Hence, ISM contributes to the insights into collective understanding of these relationships.

ISM is wildly used not only at abstraction level but also at concrete level, such as process design, strategic planning, engineering problems, product design, complex technical problems, economic development, traffic accidents, financial decision making, competitive analysis, human resources and electronic commerce [5-8]. In addition, ISM is used in improving group decision-making and knowledge management [9-11].

In ISM technique, a set of different directly and indirectly related elements are structured into a comprehensive systematic model [12]. The basic idea of ISM method is using directed graph or matrix to describe the relationship among the various known elements of the system, then compute and derive based on the matrix, eventually classify disordered elements of system into multi-level system.

\subsection{Establish Subject Level Structure Based on ISM}

\section{Establish University Financial Subject Level Structure Based on ISM}

University financial management system provides Ministry of Education with a strong support in daily management of financial decision-making. It is convenient to do all kinds of data aggregation and financial analysis through this system. It is also timely to understand the university financial situation.

University financial data warehouse index is established on the basis of enterprise financial management index, considering the nonprofit characteristics of Universities.

Step 1: Set key indexes in university financial subject data warehouse, then classify these key indicators according to decision making, these classifications of key indicators constitute the subject system, and set them to $S_{1}, S_{2}, \ldots \ldots, S_{n}$;

When setting University financial data warehouse, indicators can be divided into two groups according to their additive property. Two index sets are shown in table1 and table 2. The data warehouse contains ninety-three indicators, $I=\left\{I_{1}, I_{2}, \ldots, I_{93}\right\}$.

These ninety-three indicators can be divided into eighteen categories according to financial accounting. These eighteen categories are elements of University Financial Analysis Subject. These eighteen elements are Income structure of universities $\left(\mathrm{S}_{1}\right)$, Expenses structure of universities $\left(\mathrm{S}_{2}\right)$, Overall analysis of assets $\left(\mathrm{S}_{3}\right)$, Liabilities overall analysis $\left(\mathrm{S}_{4}\right)$, Net assets overall analysis $\left(\mathrm{S}_{5}\right)$, Human resources analysis $\left(\mathrm{S}_{6}\right)$, Educational material resources analysis $\left(\mathrm{S}_{7}\right)$, University conditions improvement $\left(\mathrm{S}_{8}\right)$, Overall income level analysis $\left(\mathrm{S}_{9}\right)$, Self-financing capacity analysis) $\left(\mathrm{S}_{10}\right)$, Operational capability analysis $\left(S_{11}\right)$, Universities profitability analysis $\left(S_{12}\right)$, University solvency ability analysis $\left(S_{13}\right)$, University development potential analysis $\left(\mathrm{S}_{14}\right)$, Human Resource utilization efficiency analysis $\left(S_{15}\right)$, Financial resources utilization efficiency analysis $\left(S_{16}\right)$, Total output effect analysis $\left(S_{17}\right)$ and Fund expenditure performance analysis $\left(S_{18}\right)$. Elements of the subject and indexes included in university financial data warehouse are as shown in table3.

$$
\mathrm{S}=\left\{\mathrm{S}_{1}, \mathrm{~S}_{2}, \mathrm{~S}_{3}, \mathrm{~S}_{4}, \mathrm{~S}_{5}, \mathrm{~S}_{6}, \mathrm{~S}_{7}, \mathrm{~S}_{8}, \mathrm{~S}_{9}, \mathrm{~S}_{10}, \mathrm{~S}_{11}, \mathrm{~S}_{12}, \mathrm{~S}_{13}, \mathrm{~S}_{14}, \mathrm{~S}_{15}, \mathrm{~S}_{16}\right. \text {, }
$$
$\left.\mathrm{S}_{17}, \mathrm{~S}_{18}\right\}$ 
Table 1. Additive University Financial Data Warehouse Index Set

\begin{tabular}{|c|c|c|c|c|c|}
\hline Cod & Index name & Code & Index name & Cod & Index name \\
\hline $\mathrm{I}_{1}$ & Funds & $\mathrm{I}_{2}$ & Central government funds & $\mathrm{I}_{3}$ & Local finance allocates funds \\
\hline $\mathrm{I}_{4}$ & Self-raised funds & $\mathrm{I}_{5}$ & Education funds & $\mathrm{I}_{6}$ & Research funds \\
\hline $\mathrm{I}_{7}$ & Total expenses & $\mathrm{I}_{8}$ & Funds expenses & $\mathrm{I}_{9}$ & Public expenses \\
\hline $\mathrm{I}_{10}$ & Education expenses & $\mathrm{I}_{11}$ & Research expenses & $I_{12}$ & Operating expenses \\
\hline $\mathrm{I}_{13}$ & $\begin{array}{l}\text { Subsidiaries expenses on ancillary } \\
\text { units }\end{array}$ & $\mathrm{I}_{14}$ & Superior Expenses & $\mathrm{I}_{15}$ & $\begin{array}{l}\text { Infrastructure construction by non- } \\
\text { financial-assistance income }\end{array}$ \\
\hline $\mathrm{I}_{16}$ & Total assets & $\mathrm{I}_{17}$ & Increase amount in assets & $\mathrm{I}_{18}$ & Total liabilities \\
\hline $\mathrm{I}_{19}$ & Increase amount in liabilities & $\mathrm{I}_{20}$ & Total net assets & $\mathrm{I}_{21}$ & Increase amount in net assets \\
\hline
\end{tabular}

Table 2. Non-additive University Financial Data Warehouse Index Set

\begin{tabular}{|c|c|c|c|c|c|}
\hline Code & Index & Code & Index & Code & Index \\
\hline $\mathrm{I}_{22}$ & Growth rate in net assets & $\mathrm{I}_{23}$ & Student-faculty ratio & $\mathrm{I}_{24}$ & Senior position ratio \\
\hline $\mathrm{I}_{25}$ & Building area per capita & $\mathrm{I}_{26}$ & Dormitory area per person & $\mathrm{I}_{27}$ & Fixed assets per capita \\
\hline $\mathrm{I}_{28}$ & Equipment cost per capita & $\mathrm{I}_{29}$ & Grants expenses per capita & $\mathrm{I}_{30}$ & Development-total expenses ratio \\
\hline $\mathrm{I}_{31}$ & Growth rate in fixed assets & $\mathrm{I}_{32}$ & Growth rate in net assets & $\mathrm{I}_{33}$ & Return on total assets \\
\hline $\mathrm{I}_{34}$ & Funds income per capita & $\mathrm{I}_{35}$ & Funds income per faculty & $\mathrm{I}_{36}$ & Total funds income growth rate \\
\hline $\mathrm{I}_{37}$ & $\begin{array}{l}\text { Financial allocation /Total funds } \\
\text { (Income) }\end{array}$ & $\mathrm{I}_{38}$ & $\begin{array}{l}\text { Education funds/Total funds } \\
\text { (Income) }\end{array}$ & $\mathrm{I}_{39}$ & $\begin{array}{l}\text { Research funds/Total funds } \\
\text { (Income) }\end{array}$ \\
\hline $\mathrm{I}_{40}$ & Self-raised -total funds income ratio & $\mathrm{I}_{41}$ & Growth rate in self-raised funds & $\mathrm{I}_{42}$ & Funds self-sufficiency rate \\
\hline $\mathrm{I}_{43}$ & Self-raised funds income per capita & $\mathrm{I}_{44}$ & Education funds/Total funds (in) & $\mathrm{I}_{45}$ & Education revenue per capita \\
\hline $\begin{array}{l}\mathrm{I}_{46} \\
\mathrm{I}_{49}\end{array}$ & $\begin{array}{l}\text { Growth rate in education revenue } \\
\text { Growth rate in research revenue }\end{array}$ & $\begin{array}{l}\mathrm{I}_{47} \\
\mathrm{I}_{50}\end{array}$ & $\begin{array}{l}\text { Research funds/Total funds (in) } \\
\text { Donation /Total funds income }\end{array}$ & $\begin{array}{l}\mathrm{I}_{48} \\
\mathrm{I}_{51}\end{array}$ & $\begin{array}{l}\text { Research funds per capita } \\
\text { Growth rate in donation income }\end{array}$ \\
\hline $\mathrm{I}_{52}$ & Funds income-expense ratio & $\mathrm{I}_{53}$ & Assets turnover & $\mathrm{I}_{54}$ & Public fund /total expenses \\
\hline $\mathrm{I}_{55}$ & Investment funds in public funds & $\mathrm{I}_{56}$ & Assets income ratio & $\mathrm{I}_{57}$ & Net assets income ratio \\
\hline $\mathrm{I}_{58}$ & Rate of return on investment & $\mathrm{I}_{59}$ & Investment income growth rate & $\mathrm{I}_{60}$ & Rate of return on investment \\
\hline $\mathrm{I}_{61}$ & Net contributes per capita & $\mathrm{I}_{62}$ & Non-financial contributes per capita & $\mathrm{I}_{63}$ & Asset-liability ratio \\
\hline $\mathrm{I}_{64}$ & $\begin{array}{l}\text { Asset-liability ratio on university-owned } \\
\text { enterprises }\end{array}$ & $\mathrm{I}_{65}$ & Income-liability ratio & $\mathrm{I}_{66}$ & Payout ratio \\
\hline $\mathrm{I}_{67}$ & Potential pay ability & $\mathrm{I}_{68}$ & Current ratio & $\mathrm{I}_{69}$ & Liability turnover \\
\hline $\mathrm{I}_{70}$ & Ratio of bank loans to total liability & $\mathrm{I}_{71}$ & Assets Equity Ratio & $\mathrm{I}_{72}$ & Utilization rate of free funds \\
\hline $\mathrm{I}_{73}$ & Utilization rate of other funds & $\mathrm{I}_{74}$ & Utilization rate of currency funds & $\mathrm{I}_{75}$ & Payout ratio in currency funds \\
\hline $\mathrm{I}_{76}$ & Ratio of net funds to currency funds & $\mathrm{I}_{77}$ & Faculty-student ratio & $\mathrm{I}_{78}$ & Student-staff ratio \\
\hline $\mathrm{I}_{79}$ & Faculty-staff ratio & $\mathrm{I}_{80}$ & Retired staff /Total staff & $\mathrm{I}_{81}$ & Personal expenses/Total expenses \\
\hline $\mathrm{I}_{82}$ & Personal-public funds expenses ratio & $\mathrm{I}_{83}$ & Public expenses per capital & $\mathrm{I}_{84}$ & Education expenses per capita \\
\hline $\mathrm{I}_{85}$ & Personal funds expenses per capita & $\mathrm{I}_{86}$ & Public funds expenses per capita & $\mathrm{I}_{87}$ & Published papers per faculty \\
\hline $\mathrm{I}_{88}$ & Published books per faculty & $\mathrm{I}_{89}$ & Research awards per faculty & $\mathrm{I}_{90}$ & Teaching awards per faculty \\
\hline $\mathrm{I}_{91}$ & Daily teaching funds expenses per capital & $\mathrm{I}_{92}$ & $\begin{array}{l}\text { Daily teaching expenses in education } \\
\text { expenses }\end{array}$ & $\mathrm{I}_{93}$ & $\begin{array}{l}\text { Proportion of daily public expenses in } \\
\text { education expenses }\end{array}$ \\
\hline
\end{tabular}

Table 3. Elements of Subject and Indexes Included in University Financial Data Warehouse

\begin{tabular}{|c|c|}
\hline Elements of subject & Indexes included \\
\hline $\mathrm{S}_{1}$ (Income structure of universities) & $\mathrm{I}_{1}, \mathrm{I}_{2}, \mathrm{I}_{3}, \mathrm{I}_{4}, \mathrm{I}_{5}, \mathrm{I}_{6}$ \\
\hline $\mathrm{S}_{2}$ (Expenses structure of universities) & $\mathrm{I}_{7}, \mathrm{I}_{8}, \mathrm{I}_{9}, \mathrm{I}_{10}, \mathbf{I}_{11}, \mathrm{I}_{12}, \mathrm{I}_{13}, \mathrm{I}_{14}, \mathrm{I}_{15}$ \\
\hline $\mathrm{S}_{3}$ (Overall analysis of assets) & $\mathrm{I}_{16} \mathrm{I}_{17}$ \\
\hline $\mathrm{S}_{4}$ (Liabilities overall analysis) & $\mathrm{I}_{18} \mathrm{I}_{19}$ \\
\hline $\mathrm{S}_{5}$ (Net assets overall analysis) & $\mathrm{I}_{20,} \mathrm{I}_{21}, \mathrm{I}_{22}$ \\
\hline $\mathrm{S}_{6}$ (Human resources analysis) & $\mathrm{I}_{23,} \mathrm{I}_{24}, 2 \mathrm{x}$ \\
\hline $\mathrm{S}_{7}$ (Educational material resources analysis) & $\mathrm{I}_{25,} \mathrm{I}_{26}, \mathrm{I}_{27}$ \\
\hline $\mathrm{S}_{8}$ (University conditions improvement) & $\mathrm{I}_{28}, \mathrm{I}_{29}, \mathrm{I}_{30}, \mathrm{I}_{31}, \mathrm{I}_{32}, \mathrm{I}_{33}$ \\
\hline $\mathrm{S}_{9}$ (Overall income level analysis) & $\mathrm{I}_{34}, \mathrm{I}_{35}, \mathrm{I}_{36}, \mathrm{I}_{37}, \mathrm{I}_{38}, \mathrm{I}_{39}$ \\
\hline $\mathrm{S}_{10}$ (Self-financing capacity analysis) & $\mathrm{I}_{40}, \mathrm{I}_{41}, \mathrm{I}_{42}, \mathrm{I}_{44}, \mathrm{I}_{46,}, \mathrm{I}_{47}, \mathrm{I}_{49}, \mathrm{I}_{50}, \mathrm{I}_{51}$ \\
\hline $\mathrm{S}_{11}$ (Operational capability analysis) & $\mathrm{I}_{52} \mathrm{I}_{53}, \mathrm{I}_{54}, \mathrm{I}_{55}$ \\
\hline $\mathrm{S}_{12}$ (Universities profitability analysis) & $\mathrm{I}_{56}, \mathrm{I}_{57}, \mathrm{I}_{58}, \mathrm{I}_{59}, \mathrm{I}_{60}, \mathrm{I}_{61}, \mathrm{I}_{62}$ \\
\hline $\mathrm{S}_{13}$ (University solvency ability analysis) & $\mathrm{I}_{63}, \mathrm{I}_{64}, \mathrm{I}_{65}, \mathrm{I}_{66}, \mathrm{I}_{67}, \mathrm{I}_{68}, \mathrm{I}_{69}, \mathrm{I}_{70}$ \\
\hline $\mathrm{S}_{14}$ (University development potential analysis) & $\mathrm{I}_{71}, \mathrm{I}_{72}, \mathrm{I}_{73}, \mathrm{I}_{74}, \mathrm{I}_{75}, \mathrm{I}_{76}$ \\
\hline $\mathrm{S}_{15}$ (Human Resource utilization efficiency analysis) & $\mathrm{I}_{77}, \mathrm{I}_{78}, \mathrm{I}_{79}, \mathrm{I}_{80}$ \\
\hline $\mathrm{S}_{16}$ (Financial resources utilization efficiency analysis) & $\mathrm{I}_{81} \mathrm{I}_{82} \mathrm{I}_{83} \mathrm{I}_{84} \mathrm{I}_{85} \mathrm{I}_{86}$ \\
\hline $\mathrm{S}_{17}$ (Total output effect analysis) & $\mathrm{I}_{87}, \mathrm{I}_{88}, \mathrm{I}_{89}, \mathrm{I}_{90}$ \\
\hline
\end{tabular}


Step 2: Establish adjacency matrix and calculate the reachability matrix

Here we define the relationship between $S_{i}$ and $S_{j}$ is that whether indexes in $S_{j}$ are used to calculate indexes in $S_{i}$. If indexes in $S_{j}$ are used to calculate indexes in $S_{i}$, then $x_{i j}$ is 1 , otherwise, $x_{i j}$ is 0 . According to this method, we established adjacency matrix A. Then we use MATLAB to calculate the reachability matrix $\mathrm{R}$ of the system by computing the adjacency matrix.

Table 4. The Adjacency Matrix for Elements of Subject in University Financial Data Warehouse

\begin{tabular}{lllllllllllllllllll}
\hline $\mathbf{A}$ & $\mathbf{1}$ & $\mathbf{2}$ & $\mathbf{3}$ & $\mathbf{4}$ & $\mathbf{5}$ & $\mathbf{6}$ & $\mathbf{7}$ & $\mathbf{8}$ & $\mathbf{9}$ & $\mathbf{1 0}$ & $\mathbf{1 1}$ & $\mathbf{1 2}$ & $\mathbf{1 3}$ & $\mathbf{1 4}$ & $\mathbf{1 5}$ & $\mathbf{1 6}$ & $\mathbf{1 7}$ & $\mathbf{1 8}$ \\
\hline $\mathbf{1}$ & 0 & 0 & 0 & 0 & 0 & 1 & 1 & 0 & 1 & 1 & 1 & 1 & 1 & 0 & 0 & 0 & 1 & 0 \\
\hline $\mathbf{2}$ & 0 & 0 & 0 & 0 & 0 & 1 & 1 & 0 & 0 & 0 & 1 & 1 & 1 & 1 & 0 & 1 & 0 & 1 \\
\hline $\mathbf{3}$ & 0 & 0 & 0 & 0 & 1 & 0 & 1 & 1 & 0 & 0 & 0 & 1 & 1 & 1 & 0 & 0 & 0 & 0 \\
\hline $\mathbf{4}$ & 0 & 0 & 0 & 0 & 1 & 0 & 0 & 0 & 0 & 0 & 0 & 0 & 1 & 0 & 0 & 0 & 0 & 0 \\
\hline $\mathbf{5}$ & 0 & 0 & 0 & 0 & 0 & 0 & 0 & 1 & 0 & 0 & 0 & 1 & 0 & 1 & 0 & 0 & 0 & 0 \\
\hline $\mathbf{6}$ & 0 & 0 & 0 & 0 & 0 & 0 & 0 & 1 & 1 & 1 & 0 & 1 & 0 & 0 & 1 & 1 & 1 & 1 \\
\hline $\mathbf{7}$ & 0 & 0 & 0 & 0 & 0 & 0 & 0 & 1 & 0 & 0 & 0 & 0 & 0 & 0 & 0 & 1 & 0 & 0 \\
\hline $\mathbf{8}$ & 0 & 0 & 0 & 0 & 0 & 0 & 0 & 0 & 0 & 0 & 1 & 0 & 0 & 0 & 0 & 0 & 0 & 0 \\
\hline $\mathbf{9}$ & 0 & 0 & 0 & 0 & 0 & 0 & 0 & 0 & 0 & 0 & 0 & 0 & 0 & 0 & 0 & 0 & 0 & 0 \\
\hline $\mathbf{1 0}$ & 0 & 0 & 0 & 0 & 0 & 0 & 0 & 0 & 0 & 0 & 1 & 0 & 0 & 0 & 0 & 0 & 0 & 0 \\
\hline $\mathbf{1 1}$ & 0 & 0 & 0 & 0 & 0 & 0 & 0 & 0 & 0 & 0 & 0 & 0 & 0 & 0 & 0 & 0 & 0 & 0 \\
\hline $\mathbf{1 2}$ & 0 & 0 & 0 & 0 & 0 & 0 & 0 & 0 & 0 & 0 & 0 & 0 & 0 & 0 & 0 & 0 & 0 & 0 \\
\hline $\mathbf{1 3}$ & 0 & 0 & 0 & 0 & 0 & 0 & 0 & 0 & 0 & 0 & 0 & 0 & 0 & 0 & 0 & 0 & 0 & 0 \\
\hline $\mathbf{1 4}$ & 0 & 0 & 0 & 0 & 0 & 0 & 0 & 0 & 0 & 0 & 0 & 0 & 0 & 0 & 0 & 0 & 0 & 0 \\
\hline $\mathbf{1 5}$ & 0 & 0 & 0 & 0 & 0 & 0 & 0 & 0 & 0 & 0 & 0 & 0 & 0 & 0 & 0 & 0 & 0 & 0 \\
\hline $\mathbf{1 6}$ & 0 & 0 & 0 & 0 & 0 & 0 & 0 & 0 & 0 & 0 & 0 & 0 & 0 & 0 & 0 & 0 & 0 & 0 \\
\hline $\mathbf{1 7}$ & 0 & 0 & 0 & 0 & 0 & 0 & 0 & 0 & 0 & 0 & 0 & 0 & 0 & 0 & 0 & 0 & 0 & 0 \\
\hline $\mathbf{1 8}$ & 0 & 0 & 0 & 0 & 0 & 0 & 0 & 0 & 0 & 0 & 0 & 0 & 0 & 0 & 0 & 0 & 0 & 0 \\
\hline
\end{tabular}

Table 5. The Reachability Matrix for Elements of Subject in University Financial Data Warehouse

\begin{tabular}{lllllllllllllllllll}
\hline $\mathbf{R}$ & $\mathbf{1}$ & $\mathbf{2}$ & $\mathbf{3}$ & $\mathbf{4}$ & $\mathbf{5}$ & $\mathbf{6}$ & $\mathbf{7}$ & $\mathbf{8}$ & $\mathbf{9}$ & $\mathbf{1 0}$ & $\mathbf{1 1}$ & $\mathbf{1 2}$ & $\mathbf{1 3}$ & $\mathbf{1 4}$ & $\mathbf{1 5}$ & $\mathbf{1 6}$ & $\mathbf{1 7}$ & $\mathbf{1 8}$ \\
\hline $\mathbf{1}$ & 1 & 0 & 0 & 0 & 0 & 1 & 1 & 1 & 1 & 1 & 1 & 1 & 1 & 0 & 1 & 1 & 1 & 1 \\
\hline $\mathbf{2}$ & 0 & 1 & 0 & 0 & 0 & 1 & 1 & 1 & 1 & 1 & 1 & 1 & 1 & 1 & 1 & 1 & 1 & 1 \\
\hline $\mathbf{3}$ & 0 & 0 & 1 & 0 & 1 & 0 & 1 & 1 & 0 & 0 & 1 & 1 & 1 & 1 & 0 & 1 & 0 & 0 \\
\hline $\mathbf{4}$ & 0 & 0 & 0 & 1 & 1 & 0 & 0 & 1 & 0 & 0 & 1 & 1 & 1 & 1 & 0 & 0 & 0 & 0 \\
\hline $\mathbf{5}$ & 0 & 0 & 0 & 0 & 1 & 0 & 0 & 1 & 0 & 0 & 1 & 1 & 0 & 1 & 0 & 0 & 0 & 0 \\
\hline $\mathbf{6}$ & 0 & 0 & 0 & 0 & 0 & 1 & 0 & 1 & 1 & 1 & 1 & 1 & 0 & 0 & 1 & 1 & 1 & 1 \\
\hline $\mathbf{7}$ & 0 & 0 & 0 & 0 & 0 & 0 & 1 & 1 & 0 & 0 & 1 & 0 & 0 & 0 & 0 & 1 & 0 & 0 \\
\hline $\mathbf{8}$ & 0 & 0 & 0 & 0 & 0 & 0 & 0 & 1 & 0 & 0 & 1 & 0 & 0 & 0 & 0 & 0 & 0 & 0 \\
\hline $\mathbf{9}$ & 0 & 0 & 0 & 0 & 0 & 0 & 0 & 0 & 1 & 0 & 0 & 0 & 0 & 0 & 0 & 0 & 0 & 0 \\
\hline $\mathbf{1 0}$ & 0 & 0 & 0 & 0 & 0 & 0 & 0 & 0 & 0 & 1 & 1 & 0 & 0 & 0 & 0 & 0 & 0 & 0 \\
\hline $\mathbf{1 1}$ & 0 & 0 & 0 & 0 & 0 & 0 & 0 & 0 & 0 & 0 & 1 & 0 & 0 & 0 & 0 & 0 & 0 & 0 \\
\hline $\mathbf{1 2}$ & 0 & 0 & 0 & 0 & 0 & 0 & 0 & 0 & 0 & 0 & 0 & 1 & 0 & 0 & 0 & 0 & 0 & 0 \\
\hline $\mathbf{1 3}$ & 0 & 0 & 0 & 0 & 0 & 0 & 0 & 0 & 0 & 0 & 0 & 0 & 1 & 0 & 0 & 0 & 0 & 0 \\
\hline $\mathbf{1 4}$ & 0 & 0 & 0 & 0 & 0 & 0 & 0 & 0 & 0 & 0 & 0 & 0 & 0 & 1 & 0 & 0 & 0 & 0 \\
\hline $\mathbf{1 5}$ & 0 & 0 & 0 & 0 & 0 & 0 & 0 & 0 & 0 & 0 & 0 & 0 & 0 & 0 & 1 & 0 & 0 & 0 \\
\hline $\mathbf{1 6}$ & 0 & 0 & 0 & 0 & 0 & 0 & 0 & 0 & 0 & 0 & 0 & 0 & 0 & 0 & 0 & 1 & 0 & 0 \\
\hline $\mathbf{1 7}$ & 0 & 0 & 0 & 0 & 0 & 0 & 0 & 0 & 0 & 0 & 0 & 0 & 0 & 0 & 0 & 0 & 1 & 0 \\
\hline $\mathbf{1 8}$ & 0 & 0 & 0 & 0 & 0 & 0 & 0 & 0 & 0 & 0 & 0 & 0 & 0 & 0 & 0 & 0 & 0 & 1 \\
\hline
\end{tabular}


Step 3: Derive reachability set and antecedent sets from the final reachability and establish level structure.

The top level $\left(\mathrm{L}_{1}\right)$ is shown in table 6 . This process is continued until the level of each element is found. The results of derived levels are as fellows.

Table 6. The Top Level Elements Set $\left(L_{1}\right)$ Analysis

\begin{tabular}{|c|c|c|c|}
\hline $\mathrm{S}_{\mathrm{i}}$ & $\mathrm{R}\left(\mathrm{S}_{\mathrm{i}}\right)$ & $\mathrm{A}\left(\mathrm{S}_{\mathrm{i}}\right)$ & $\mathrm{R}\left(\mathrm{S}_{\mathrm{i}}\right) \cap \mathrm{A}\left(\mathrm{S}_{\mathrm{i}}\right)$ \\
\hline$S_{1}$ & $\mathrm{~S}_{1}, \mathrm{~S}_{6}, \mathrm{~S}_{7}, \mathrm{~S}_{8}, \mathrm{~S}_{9}, \mathrm{~S}_{10,}, \mathrm{~S}_{11}, \mathrm{~S}_{12}, \mathrm{~S}_{15}, \mathrm{~S}_{16,} \mathrm{~S}_{17}, \mathrm{~S}_{18}$ & $\mathrm{~S}_{1}$ & $\mathrm{~S}_{1}$ \\
\hline $\mathrm{S}_{2}$ & $\mathrm{~S}_{2}, \mathrm{~S}_{6}, \mathrm{~S}_{7}, \mathrm{~S}_{8}, \mathrm{~S}_{9,} \mathrm{~S}_{10,} \mathrm{~S}_{11,} \mathrm{~S}_{12}, \mathrm{~S}_{14}, \mathrm{~S}_{15}, \mathrm{~S}_{16,} \mathrm{~S}_{17} \mathrm{~S}_{18}$ & $\mathrm{~S}_{2}$ & $\mathrm{~S}_{2}$ \\
\hline $\mathrm{S}_{3}$ & $\mathrm{~S}_{3}, \mathrm{~S}_{5}, \mathrm{~S}_{7}, \mathrm{~S}_{8}, \mathrm{~S}_{11}, \mathrm{~S}_{12}, \mathrm{~S}_{13}, \mathrm{~S}_{14}, \mathrm{~S}_{16}$ & $\mathrm{~S}_{3}$ & $\mathrm{~S}_{3}$ \\
\hline $\mathrm{S}_{4}$ & $\mathrm{~S}_{4}, \mathrm{~S}_{5}, \mathrm{~S}_{7}, \mathrm{~S}_{8}, \mathrm{~S}_{11}, \mathrm{~S}_{12}, \mathrm{~S}_{13}, \mathrm{~S}_{14}, \mathrm{~S}_{16}$ & $\mathrm{~S}_{4}$ & $\mathrm{~S}_{4}$ \\
\hline $\mathrm{S}_{5}$ & $\mathrm{~S}_{5,} \mathrm{~S}_{8,} \mathrm{~S}_{11} \mathrm{~S}_{12} \mathrm{~S}_{14}$ & $\mathrm{~S}_{3,} \mathrm{~S}_{4} \mathrm{~S}_{5}$ & $\mathrm{~S}_{5}$ \\
\hline $\mathrm{S}_{6}$ & $\mathrm{~S}_{6}, \mathrm{~S}_{8,} \mathrm{~S}_{9,} \mathrm{~S}_{10,} \mathrm{~S}_{11}, \mathrm{~S}_{12}, \mathrm{~S}_{15}, \mathrm{~S}_{16}, \mathrm{~S}_{17} \mathrm{~S}_{18}$ & $\mathrm{~S}_{1}, \mathrm{~S}_{2}, \mathrm{~S}_{6}$ & $\mathrm{~S}_{6}$ \\
\hline $\mathrm{S}_{7}$ & $\mathrm{~S}_{7}, \mathrm{~S}_{8}, \mathrm{~S}_{11}, \mathrm{~S}_{16}$ & $\mathrm{~S}_{1} \mathrm{~S}_{2}, \mathrm{~S}_{3}, \mathrm{~S}_{7}$ & $\mathrm{~S}_{7}$ \\
\hline $\mathrm{S}_{8}$ & $\mathrm{~S}_{8}, \mathrm{~S}_{11}$ & $\mathrm{~S}_{1}, \mathrm{~S}_{2}, \mathrm{~S}_{3}, \mathrm{~S}_{4}, \mathrm{~S}_{5}, \mathrm{~S}_{6}, \mathrm{~S}_{7}, \mathrm{~S}_{8}$ & $\mathrm{~S}_{8}$ \\
\hline $\mathrm{S}_{9}$ & $\mathrm{~S}_{9}$ & $\mathrm{~S}_{1}, \mathrm{~S}_{2}, \mathrm{~S}_{6}, \mathrm{~S}_{9}$ & $\mathrm{~S}_{9}$ \\
\hline $\mathrm{S}_{10}$ & $\mathrm{~S}_{10,} \mathrm{~S}_{11}$ & $\mathrm{~S}_{1}, \mathrm{~S}_{2}, \mathrm{~S}_{6}, \mathrm{~S}_{10}$ & $\mathrm{~S}_{10}$ \\
\hline$S_{11}$ & $\mathrm{~S}_{11}$ & $\mathrm{~S}_{1}, \mathrm{~S}_{2}, \mathrm{~S}_{3}, \mathrm{~S}_{4}, \mathrm{~S}_{5}, \mathrm{~S}_{6}, \mathrm{~S}_{7}, \mathrm{~S}_{8}, \mathrm{~S}_{9}, \mathrm{~S}_{10}, \mathrm{~S}_{11}$ & $\mathrm{~S}_{11}$ \\
\hline$S_{12}$ & $\mathrm{~S}_{12}$ & $\mathrm{~S}_{1}, \mathrm{~S}_{2}, \mathrm{~S}_{3}, \mathrm{~S}_{4}, \mathrm{~S}_{5}, \mathrm{~S}_{6}, \mathrm{~S}_{12}$ & $\mathrm{~S}_{12}$ \\
\hline$S_{13}$ & $S_{13}$ & $\mathrm{~S}_{1}, \mathrm{~S}_{2}, \mathrm{~S}_{3}, \mathrm{~S}_{4}, \mathrm{~S}_{13}$ & $\mathrm{~S}_{13}$ \\
\hline $\mathrm{S}_{14}$ & $\mathrm{~S}_{14}$ & $\mathrm{~S}_{2}, \mathrm{~S}_{3}, \mathrm{~S}_{4}, \mathrm{~S}_{5}, \mathrm{~S}_{14}$ & $\mathrm{~S}_{14}$ \\
\hline$S_{15}$ & $\mathrm{~S}_{15}$ & $\mathrm{~S}_{1}, \mathrm{~S}_{2}, \mathrm{~S}_{6}, \mathrm{~S}_{15}$ & $\mathrm{~S}_{15}$ \\
\hline$S_{16}$ & $\mathrm{~S}_{16}$ & $\mathrm{~S}_{1}, \mathrm{~S}_{2}, \mathrm{~S}_{3}, \mathrm{~S}_{6}, \mathrm{~S}_{7}, \mathrm{~S}_{16}$ & $\mathrm{~S}_{16}$ \\
\hline$S_{17}$ & $S_{17}$ & $\mathrm{~S}_{1}, \mathrm{~S}_{2}, \mathrm{~S}_{6}, \mathrm{~S}_{17}$ & $S_{17}$ \\
\hline $\mathrm{S}_{18}$ & $\mathrm{~S}_{18}$ & $\mathrm{~S}_{1}, \mathrm{~S}_{2}, \mathrm{~S}_{6}, \mathrm{~S}_{18}$ & $\mathrm{~S}_{18}$ \\
\hline
\end{tabular}

From table $6, \mathrm{~L}_{1}$ is derived. $\mathrm{L}_{1}=\left\{\mathrm{S}_{9}, \mathrm{~S}_{11}, \mathrm{~S}_{12}, \mathrm{~S}_{13}, \mathrm{~S}_{14}, \mathrm{~S}_{15}, \mathrm{~S}_{16}, \mathrm{~S}_{17}, \mathrm{~S}_{18}\right\}$;

Table 7. The Second Level Elements Set $\left(L_{2}\right)$ Analysis

\begin{tabular}{llll}
\hline $\mathrm{S}_{\mathrm{i}}$ & \multicolumn{1}{c}{$\mathrm{R}\left(\mathrm{S}_{\mathrm{i}}\right)$} & \multicolumn{1}{c}{$\mathrm{A}\left(\mathrm{S}_{\mathrm{i}}\right)$} & $\mathrm{R}\left(\mathrm{S}_{\mathrm{i}}\right) \cap \mathrm{A}\left(\mathrm{S}_{\mathrm{i}}\right)$ \\
\hline $\mathrm{S}_{1}$ & $\mathrm{~S}_{1}, \mathrm{~S}_{6}, \mathrm{~S}_{7}, \mathrm{~S}_{8}, \mathrm{~S}_{10}$ & $\mathrm{~S}_{1}$ & $\mathrm{~S}_{1}$ \\
$\mathrm{~S}_{2}$ & $\mathrm{~S}_{2}, \mathrm{~S}_{6}, \mathrm{~S}_{7}, \mathrm{~S}_{8}, \mathrm{~S}_{10}$ & $\mathrm{~S}_{2}$ & $\mathrm{~S}_{2}$ \\
$\mathrm{~S}_{3}$ & $\mathrm{~S}_{3}, \mathrm{~S}_{5}, \mathrm{~S}_{7}, \mathrm{~S}_{8}$ & $\mathrm{~S}_{3}$ & $\mathrm{~S}_{3}$ \\
$\mathrm{~S}_{4}$ & $\mathrm{~S}_{4}, \mathrm{~S}_{5}, \mathrm{~S}_{7}, \mathrm{~S}_{8}$ & $\mathrm{~S}_{4}$ & $\mathrm{~S}_{4}$ \\
$\mathrm{~S}_{5}$ & $\mathrm{~S}_{5}, \mathrm{~S}_{8}$ & $\mathrm{~S}_{3}, \mathrm{~S}_{4}, \mathrm{~S}_{5}$ & $\mathrm{~S}_{5}$ \\
$\mathrm{~S}_{6}$ & $\mathrm{~S}_{6} \mathrm{~S}_{8}, \mathrm{~S}_{10}$ & $\mathrm{~S}_{1}, \mathrm{~S}_{2}, \mathrm{~S}_{6}$ & $\mathrm{~S}_{6}$ \\
$\mathrm{~S}_{7}$ & $\mathrm{~S}_{7}, \mathrm{~S}_{8}$ & $\mathrm{~S}_{1}, \mathrm{~S}_{2}, \mathrm{~S}_{3}, \mathrm{~S}_{7}$ & $\mathrm{~S}_{7}$ \\
$\mathrm{~S}_{8}$ & $\mathrm{~S}_{8}$ & $\mathrm{~S}_{1}, \mathrm{~S}_{2}, \mathrm{~S}_{3}, \mathrm{~S}_{4}, \mathrm{~S}_{5}, \mathrm{~S}_{6}, \mathrm{~S}_{7}, \mathrm{~S}_{8}$ & $\mathrm{~S}_{8}$ \\
$\mathrm{~S}_{10}$ & $\mathrm{~S}_{10}$ & $\mathrm{~S}_{1}, \mathrm{~S}_{2}, \mathrm{~S}_{6}, \mathrm{~S}_{10}$ & $\mathrm{~S}_{10}$ \\
\hline
\end{tabular}

From table $7, \mathrm{~L}_{2}$ is derived. $\mathrm{L}_{2}=\left\{\mathrm{S}_{8}, \mathrm{~S}_{10}\right\}$; 
Table 8. The Third Level Elements Set $\left(L_{3}\right)$ Analysis

\begin{tabular}{lllc}
\hline $\mathrm{S}_{\mathrm{i}}$ & \multicolumn{1}{c}{$\mathrm{R}\left(\mathrm{S}_{\mathrm{i}}\right)$} & \multicolumn{1}{c}{$\mathrm{A}\left(\mathrm{S}_{\mathrm{i}}\right)$} & $\mathrm{R}\left(\mathrm{S}_{\mathrm{i}}\right) \cap \mathrm{A}\left(\mathrm{S}_{\mathrm{i}}\right)$ \\
\hline $\mathrm{S}_{1}$ & $\mathrm{~S}_{1}, \mathrm{~S}_{6}, \mathrm{~S}_{7}$ & $\mathrm{~S}_{1}$ & $\mathrm{~S}_{1}$ \\
$\mathrm{~S}_{2}$ & $\mathrm{~S}_{2}, \mathrm{~S}_{6}, \mathrm{~S}_{7}$ & $\mathrm{~S}_{2}$ & $\mathrm{~S}_{2}$ \\
$\mathrm{~S}_{3}$ & $\mathrm{~S}_{3}, \mathrm{~S}_{5}, \mathrm{~S}_{7}$ & $\mathrm{~S}_{3}$ & $\mathrm{~S}_{3}$ \\
$\mathrm{~S}_{4}$ & $\mathrm{~S}_{4}, \mathrm{~S}_{5}, \mathrm{~S}_{7}$ & $\mathrm{~S}_{4}$ & $\mathrm{~S}_{4}$ \\
$\mathrm{~S}_{5}$ & $\mathrm{~S}_{5}$ & $\mathrm{~S}_{3}, \mathrm{~S}_{4}, \mathrm{~S}_{5}$ & $\mathrm{~S}_{5}$ \\
$\mathrm{~S}_{6}$ & $\mathrm{~S}_{6}$ & $\mathrm{~S}_{1}, \mathrm{~S}_{2}, \mathrm{~S}_{6}$ & $\mathrm{~S}_{6}$ \\
$\mathrm{~S}_{7}$ & $\mathrm{~S}_{7}$ & $\mathrm{~S}_{1}, \mathrm{~S}_{2}, \mathrm{~S}_{3}, \mathrm{~S}_{7}$ & $\mathrm{~S}_{7}$ \\
\hline
\end{tabular}

From table $8, \mathrm{~L}_{3}$ is derived. $\mathrm{L}_{3}=\left\{\mathrm{S}_{5}, \mathrm{~S}_{6}, \mathrm{~S}_{7}\right\}$;

Table 9. The Fourth Level Elements Set $\left(\mathrm{L}_{4}\right)$ Analysis

\begin{tabular}{cccc}
\hline $\mathrm{S}_{\mathrm{i}}$ & $\mathrm{R}\left(\mathrm{S}_{\mathrm{i}}\right)$ & $\mathrm{A}\left(\mathrm{S}_{\mathrm{i}}\right)$ & $\mathrm{R}\left(\mathrm{S}_{\mathrm{i}}\right) \cap \mathrm{A}\left(\mathrm{S}_{\mathrm{i}}\right)$ \\
\hline $\mathrm{S}_{1}$ & $\mathrm{~S}_{1}$ & $\mathrm{~S}_{1}$ & $\mathrm{~S}_{1}$ \\
$\mathrm{~S}_{2}$ & $\mathrm{~S}_{2}$ & $\mathrm{~S}_{2}$ & $\mathrm{~S}_{2}$ \\
$\mathrm{~S}_{3}$ & $\mathrm{~S}_{3}$ & $\mathrm{~S}_{3}$ & $\mathrm{~S}_{3}$ \\
$\mathrm{~S}_{4}$ & $\mathrm{~S}_{4}$ & $\mathrm{~S}_{4}$ & $\mathrm{~S}_{4}$ \\
\hline
\end{tabular}

From table 9, $\mathrm{L}_{4}$ is derived. $\mathrm{L}_{4}=\left\{\mathrm{S}_{1}, \mathrm{~S}_{2}, \mathrm{~S}_{3}, \mathrm{~S}_{4}\right\}$;

Step 5: Set the bottom level $L_{4}$ and remaining level $L_{1}, L_{2}, L_{3}$ to basic level $L_{b}$ and analysis level $L_{a}$ in the system level structure. The top level $L_{1}$ is set to the value level $L_{v}$. Then establish a subject-level model.

Table 10. The University Financial Data Warehouse Subject Levels

\begin{tabular}{lll}
\hline Subject Level & Code of subject elements & Name of the subject elements \\
\hline $\mathrm{L}_{\mathrm{b}}$ & $\mathrm{S}_{1}$ & Income structure of universities \\
\cline { 2 - 3 } & $\mathrm{S}_{2}$ & Expenses structure of universities \\
\cline { 2 - 3 } & $\mathrm{S}_{3}$ & Overall analysis of assets \\
\cline { 2 - 3 } & $\mathrm{S}_{4}$ & Liabilities overall analysis \\
\hline $\mathrm{L}_{\mathrm{a}}$ & $\mathrm{S}_{5}$ & Net assets overall analysis \\
\cline { 2 - 3 } & $\mathrm{S}_{6}$ & Human resources analysis \\
\cline { 2 - 3 } & $\mathrm{S}_{7}$ & Educational material resources analysis \\
\cline { 2 - 3 } & $\mathrm{S}_{8}$ & University conditions improvement \\
\cline { 2 - 3 } & $\mathrm{S}_{10}$ & Self-financing capacity analysis \\
\cline { 2 - 3 } $\mathrm{L}_{\mathrm{v}}$ & $\mathrm{S}_{9}$ & Overall income level analysis \\
\cline { 2 - 3 } & $\mathrm{S}_{11}$ & Operational capability analysis \\
\cline { 2 - 3 } & $\mathrm{S}_{12}$ & Universities profitability analysis \\
\cline { 2 - 3 } & $\mathrm{S}_{13}$ & University solvency ability analysis \\
\cline { 2 - 3 } & $\mathrm{S}_{14}$ & University development potential analysis \\
\cline { 2 - 3 } & $\mathrm{S}_{15}$ & Human Resource utilization efficiency analysis \\
\cline { 2 - 3 } & $\mathrm{S}_{16}$ & Tonancial resources utilization efficiency analysis \\
\cline { 2 - 3 } & $\mathrm{S}_{17}$ & Fund expenditure performance analysis \\
\cline { 2 - 3 } & $\mathrm{S}_{18}$ & \\
\hline
\end{tabular}




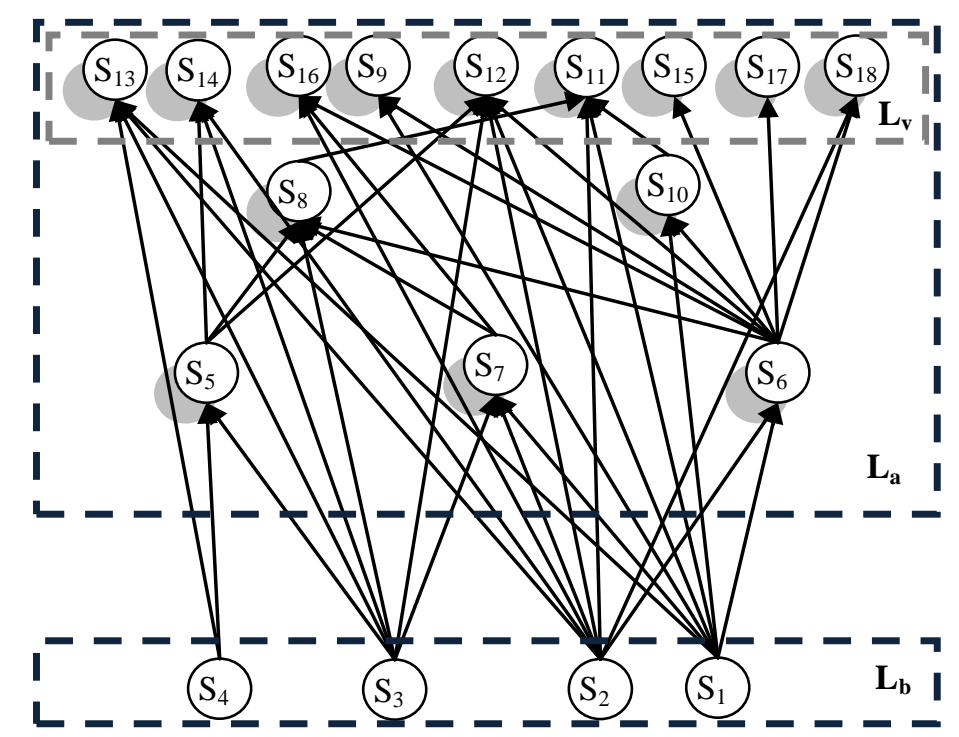

Figure 1. Level Structure of University Financial Analysis Subject based on ISM

As it is shown in Fig.1.We finally obtain hierarchy structure of University Financial Analysis Subject based on Interpretative Structure Modeling. Basic level of the University Financial Analysis Subject consists four elements (Income structure of universities, Expenses structure of universities, Overall analysis of assets and Liabilities overall analysis) that are most fundamental among those elements. This result coincides with knowledge of financial management. Income and expenses are significance component in income statement that is a direct result of the information that transformed recorded in the journals and ledgers into concise, compiled revenue and expense figures to be used by management. Assets and liabilities as remarkable parts in balance sheet are also fundamental to managers of university in reality. Because net worth of the business that is the difference between the assets and the liabilities is a measurement of the time the business is expected to stay in financial power. Moreover, It also provides the business with information on how best it is able to pay its debts. Hence, this Level structure of University Financial Analysis Subject is not conflict with the real financial management. The important feature of this structure is that it is established from the perspective of hierarchy system modeling and university financial requirements.

\section{Conclusion}

Elements of subject are divided into base level and analysis level. There is a special level named value level, which is a collection of elements that provide decision makers with most valuable information. This paper presents a general method based on ISM to establish subject level structure in data warehouse. The advantage of this method is that it introduces quantitative tools, such as directed graph, adjacency matrix and reachability matrix to partition subject level in data warehouse. A case on university financial data warehouse's subject level structure establishment verifies the rationality and validity of the method. This method gives a new idea for establishing data warehouse's subject level.

\section{Acknowledgments}

The research is supported by national natural science fund of China (71272161). 


\section{References}

[1] D. R. Farris and A. P. Sage, "On the use of interpretive structural modeling for worth assessment", Computer and Electrical Engineering, vol.2, (1975), pp.149-174.

A. P. Sage, Interpretive structural modeling. Methodology for large scale systems, McGraw-Hill, New York, (1977), pp.91-164.

[2] T. Raj, R. Shankar and M. Suhaib, An ISM approach for modeling the enablers of flexible manufacturing system: The case for India. International Journal of Production Research, vol.46, no.24, (2007), pp.1-30.

[3] M. D. Singh, R. Shankar, R. Narain and A. Agarwal, "An interpretive structural modeling of knowledge management in engineering industries", Journal of Advances in Management Research, vol.1, no.1, (2003), pp.28-40.

[4] S. Chidambaranathan, C. Muralidharan and S. G. Deshmukh, "Analyzing the interaction of critical factors of supplier development using Interpretive Structural Modeling-an empirical study", International Journal of Advance Manufacturing Technology, vol.43, (2009), pp.1081-1093.

[5] W. L. Li, P. Humphreys, L. Y. Chan and M. Kumaraswamy, "Predicting purchasing performance: the role of supplier development programs", Journal of Material Processing Technology, vol.138, no.1-3, (2003), pp.243-249.

[6] D. K. Banwet and R. Arora, "Enablers and inhibitors of ecommerce implementation in India-an interpretive structural modeling (ISM) approach”, In: Kanda A et al (ed) Operations management for global economy challenges and prospects, Phoenix, New Delhi, (1999), pp.332-341.

[7] K. S. Rajesh, K. G. Suresh and S. G. Deshmukh, "Interpretive structural modeling of factors for improving competitiveness of SMEs", International Journal of Productivity and Quality Management, vol.2, no.4, (2007), pp.423-440.

[8] R. S. Tabrizi, Y. P. Foong and N. Ebrahimi, "Using Interpretive Structural Modeling to Determine the Relationships among Knowledge Management Criteria inside Malaysian Organizations", World Academy of Science, Engineering and Technology, vol.48, (2010), pp.727-732.

[9] M. D. Singh and R. Kant, "Knowledge management barriers: An interpretive structural modeling approach", International Journal of Management Science and Engineering Management, vol.3, no.2, (2008), pp.141-150.

[10] R. Bolaños, E. Fontela, A. Nenclares and P. Pastor, "Using interpretive structural modelling in strategic decision-making groups", Management Decision, vol.43, no.6, (2005), pp.877 - 895.

[11] J. N. Warfield, "Developing Subsystem Matrices in Structural Modeling", IEEE Transaction System, Man, and Cybernetics, vol.4, no.1, (1974), pp.74-81. 